\title{
Progressão harmônica e o triângulo de Leibniz
}

\author{
Harmonic progression and the triangle of Leibniz
}

\author{
David Pinto Martins ${ }^{1}$ \\ ${ }^{1}$ Colégio Estadual Pinto de Aguiar, Secretaria de Educação da Bahia. BA, Brasil \\ oprofessordavid@yahoo.com.br
}

\begin{abstract}
Resumo
Este artigo pretende abordar de uma forma elementar o estudo das progressões harmônicas. Para este fim, o uso da história da matemática e estratégias de resolução de problemas permearam o texto. Vários problemas, alguns clássicos e outros extraídos de olimpíadas de matemática, foram tratados para mostrar a ampla aplicabilidade deste assunto. No final, o triângulo de Leibniz e sua relação com as progressões harmônicas é estudado.
\end{abstract}

Palavras-chave: Progressão harmônica; História da matemática; Resolução de problemas; Triângulo de Leibniz.

\begin{abstract}
This article intends to address in an elementary way the study of harmonic progressions. To this end, the usage of history of mathematics and problem solving strategies permeated the text. Several problems, some classics and other extracted from mathematical olympiads, were treated to show the wide applicability of this subject. In the end, the triangle of Leibniz and his relationship with the harmonic progressions is studied.
\end{abstract}

Keywords: Harmonic progression, History of mathematics, Problem solving, Triangle of Leibniz. 


\section{Introdução}

O estudo das progressões na maioria das escolas brasileiras limita-se tão somente a analisar as progressões aritméticas e geométricas, em um roteiro quase sempre definido da seguinte forma: primeiro, se estuda a progressão aritmética, o que essencialmente significa obter a expressão do termo geral da progressão aritmética e a soma dos seus termos, além da resolução de problemas, contextualizados ou não; e depois, a progressão geométrica, que basicamente segue o mesmo caminho da progressão aritmética, com a particularidade de que aqui se determina também a soma de infinitos termos, quando a razão desta é um número do intervalo $(-1 ; 1)$.

Progressões, quase sempre, não estão, por parte dos estudantes, entre os assuntos mais temidos de matemática. Entretanto, um aluno mais curioso pode se perguntar se estas seriam as únicas progressões existentes; e, existindo outra progressão, como ela é e o que é possível fazer com tal.

A realidade é que mesmo licenciados em matemática revelam em sua maioria um desconhecimento do tema, se limitando a reproduzirem o que dizem os livros para o ensino médio de matemática sobre os assuntos. Neste artigo, o leitor terá a possibilidade de resolver problemas que, até então, não teria condições de resolver com a matemática vista apenas nas progressões aritmética e geométrica.

Aqui, abordamos expositivamente, na teoria e na prática, com o uso de história da matemática e com ênfase na resolução de problemas, a progressão harmônica. $\mathrm{O}$ desenvolvimento deste conteúdo permitirá abordar o triângulo de Leibniz, ferramenta matemática que permitiu ao grande matemático alemão calcular suas primeiras séries.

Conforme dito antes, a ênfase na resolução de problemas como agente motivador do ensino em matemática está presente neste artigo, por ser uma das melhores formas de se promover a real aprendizagem matemática para os alunos. Neste sentido, o notável matemático húngaro George Polya (1887 - 1985) deixou uma lição muito conhecida no meio matemático, quando dividiu o processo de resolução de problemas em matemática em quatro passos básicos. O primeiro passo é a compreensão do problema; em seguida, a construção de uma estratégia de resolução; o penúltimo passo é a execução da estratégia e, por fim, a revisão da solução. Polya sabia da importância de se resolver problemas em matemática para a consistência da aprendizagem de quem a estuda.

A realização das Olimpíadas de Matemática, sobretudo a OBMEP (Olimpíada Brasileira de Matemática da Escola Pública), recentemente criada no Brasil, é uma medida positiva para o aumento do interesse do estudo da matemática e reforça a posição de que a resolução de problemas em matemática é fundamental para o conhecimento da mesma. Muito da teoria e dos problemas aqui vistos se encaixam neste espírito olímpico, de raciocínio diferenciado.

Despertar o interesse para tais contéudos, além de enriquecedor matematicamente, permite que se questione se outros temas, até então inacessíveis com a matemática básica, podem ser expandidos. Estes temas podem ser desenvolvidos, em alguns casos, sem o uso da matemática vista no nível superior, o que pode ser estimulante ao espírito pesquisador - o que é muito bom de se instigar em quem estuda.

\section{Ideias iniciais}

Uma progressão harmônica é uma sequência finita ou infinita de números cujos termos são todos diferentes de zero e tais que seus inversos formam uma progressão aritmética de primeira ordem.

Assim, a sequência $\left(a_{1}, a_{2}, \ldots, a_{n}, \ldots\right)$ é uma progressão harmônica se, e somente se, a sequência dada por $\left(\frac{1}{a_{1}}, \frac{1}{a_{2}}, \ldots, \frac{1}{a_{n}}, \ldots\right)$ é uma progressão aritmética de primeira ordem.

Definimos a progressão harmônica finita de modo inteiramente análogo. Para simplificar, daqui por diante denominaremos a progressão aritmética de primeira ordem que está associada a uma dada progressão harmônica apenas de progressão aritmética, seja ela finita ou infinita.

Determinemos a expressão do termo geral de uma progressão harmônica. Para tanto, seja $a_{j}$ um termo qualquer da progressão harmônica $\left(a_{n}\right)$, então $b_{j}=\frac{1}{a_{j}}$ é o termo geral de uma progressão aritmética de razão $r$ tal que

$$
r=b_{2}-b_{1}=\frac{1}{a_{2}}-\frac{1}{a_{1}}=\frac{a_{1}-a_{2}}{a_{1} a_{2}}
$$

e então temos que

$$
b_{j}=b_{1}+(j-1) r=\frac{a_{2}+(j-1)\left(a_{1}-a_{2}\right)}{a_{1} a_{2}} .
$$

Como $b_{j}=\frac{1}{a_{j}}$, segue que

$$
\frac{1}{a_{j}}=\frac{a_{2}+(j-1)\left(a_{1}-a_{2}\right)}{a_{1} a_{2}}
$$

e assim obtemos

$$
a_{j}=\frac{a_{1} a_{2}}{a_{2}+(j-1)\left(a_{1}-a_{2}\right)}
$$

que é a expressão do termo geral de uma progressão harmônica para $j>2$, onde $j$ é um número natural.

São exemplos de progressões harmônicas as sequências $\left(a_{n}\right),\left(b_{n}\right)$ e $\left(c_{n}\right)$ dispostas a seguir:

$$
\left(a_{n}\right)=\left(1, \frac{1}{2}, \frac{1}{3}, \frac{1}{4}, \ldots, \frac{1}{n}, \ldots\right)
$$




$$
\begin{gathered}
\left(b_{n}\right)=\left(\frac{1}{5}, \frac{1}{2},-1,-\frac{1}{4},-\frac{1}{7}\right) \\
\left(c_{n}\right)=(4,4,4,4, \ldots)
\end{gathered}
$$

As sequências $\left(a_{n}\right),\left(b_{n}\right)$ e $\left(c_{n}\right)$ são progressões harmônicas pois podemos associar, respectivamente, à $\left(a_{n}\right),\left(b_{n}\right)$ e $\left(c_{n}\right)$ as sequências

$$
\begin{gathered}
\left(d_{n}\right)=(1,2, \ldots, n, \ldots) \\
\left(e_{n}\right)=(5,2,-1,-4,-7)
\end{gathered}
$$

e

$$
\left(f_{n}\right)=\left(\frac{1}{4}, \frac{1}{4}, \frac{1}{4}, \frac{1}{4}, \ldots\right),
$$

que são progressões aritméticas.

É possível classificar as progressões harmônicas quanto ao número de termos: uma progressão harmônica pode ser finita, como por exemplo a progressão harmônica $\left(b_{n}\right)$ acima, ou infinita, como a progressão harmônica $\left(a_{n}\right)$ anterior.

Problema 1. Dada a progressão harmônica $\left(3, \frac{12}{7}, \frac{6}{5}, \ldots\right)$, determine o seu nono termo.

Solução. Temos pela igualdade 1 que

$$
a_{9}=\frac{\left(3 \cdot \frac{12}{7}\right)}{\frac{12}{7}+(9-1)\left(3-\frac{12}{7}\right)}=\frac{3}{7} \text {. }
$$

Muito útil será a definição de média harmônica. Os gregos antigos a estudaram, gerando problemas e descobertas interessantes, que aqui serão abordados. A definição de média harmônica disposta a seguir, para três números, pode ser facilmente generalizada para $k$ números, onde $k$ é um inteiro positivo qualquer: dados três números $a, H, b$, dizemos que $H$ é a média harmônica de $a$ e $b$ se entre estes números vale a igualdade $\frac{1}{a}+\frac{1}{b}=\frac{1}{H}+\frac{1}{H}$, que é equivalente à igualdade $H=\frac{2 a b}{a+b}$.

Percebemos então desta definição que o significado de média harmônica entre dois números é obter um número $H$ tal que a soma do inverso de $a$ com o inverso de $b$ seja igual à soma do inverso de $H$ consigo próprio; ou seja, podemos substituir cada uma das frações $\frac{1}{a}$ e $\frac{1}{b}$ por $\frac{1}{H}$ que o resultado da soma entre eles é preservado.

Na Grécia antiga, cerca de 500 anos antes de Cristo, uma corrente filosófica marcou época. Os pitagóricos eram uma escola de pensadores cujo líder foi o matemático e filósofo Pitágoras, o qual acredita-se ter nascido em 565 a.C. e falecido em 490 a.C.. Os pitagóricos em muito contribuíram para a matemática e a cultura de modo geral.

Os pitagóricos definiam a média harmônica assim: dados dois números positivos $a$ e $b, H$ é a média harmônica de $a$ e $b$ se existe um número $n$ tal que $a=H+\frac{a}{n}$ e
$H=b+\frac{b}{n}$. Esta definição é equivalente à definição de média harmônica, pois ao multiplicarmos por $b$ a igualdade $a=H+\frac{a}{n}$ temos que

$$
a b=b H+\frac{a b}{n}
$$

e assim

$$
a b-b H=\frac{a b}{n} .
$$

Analogamente, quando multiplicamos por $a$ a igualdade $H=b+\frac{b}{n}$ temos

$$
a H=a b+\frac{a b}{n}
$$

e assim

$$
a b-a H=-\frac{a b}{n} .
$$

Somando membro a membro estas duas últimas igualdades, temos:

$$
\begin{gathered}
2 a b-b H-a H=0 \\
2 a b-H(b+a)=0 \\
2 a b=H(a+b)
\end{gathered}
$$

e portanto

$$
H=\frac{2 a b}{a+b}
$$

\section{Uma propriedade das progressões harmônicas e a harmonia geomé- trica de Filolao}

Uma propriedade que vale para as progressões harmônicas é a seguinte: em toda progressão harmônica, quaisquer três termos consecutivos são tais que o segundo é a média harmônica dos outros dois. Provemos essa assertiva:

Demonstração. Sejam $a, h, b$ três termos de uma progressão harmônica. Por definição, temos que $\left(\frac{1}{a}, \frac{1}{h}, \frac{1}{b}\right)$ é uma progressão aritmética. Logo, vale a igualdade

$$
\frac{1}{h}=\frac{\frac{1}{a}+\frac{1}{b}}{2} \text {. }
$$

Assim, segue que

$$
\frac{1}{h}=\frac{\frac{b+a}{a b}}{2}=\frac{a+b}{2 a b}
$$

$\log 0$

$$
h=\frac{2 a b}{a+b} .
$$




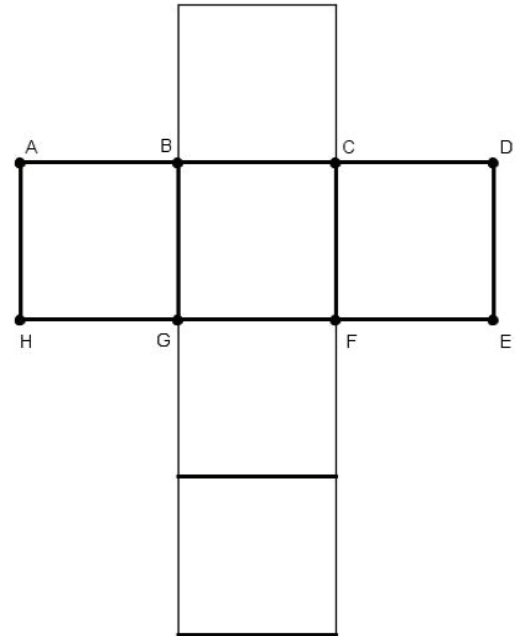

Figura 1: A harmonia geométrica do cubo.

De posse dessa propriedade, podemos resolver o problema a seguir, que explica o motivo de Filolao, um pitagórico que viveu por volta de 425 a.C., denominar o cubo de harmonia geométrica.

Problema 2. Determine o segundo termo de uma progressão harmônica $\left(a_{n}\right)$ cujo primeiro termo é igual a 6 e tal que $a_{3}=2 a_{1}$.

Solução. Temos $a_{3}=12 \mathrm{e}$, pela propriedade das progressões harmônicas vista anteriormente, $a_{2}$ é a média harmônica entre os inteiros positivos $a_{1}=6$ e $a_{3}=12$. Assim, segue que:

$$
a_{2}=\frac{2 \cdot 6 \cdot 12}{6+12}=\frac{144}{18}=8 .
$$

O motivo de Filolao chamar o cubo de harmonia geométrica se deve ao fato de que este tem 6 faces, 8 vértices e 12 arestas e, pelo resultado do último problema, vemos que o número de vértices de um cubo, que é uma figura geométrica espacial, é a média harmônica entre o seu número de faces e o seu número de arestas. A figura 1 é de um cubo planificado.

As seis faces são os quadrados, os oito vértices são os pontos denotados por letras maiúsculas (os demais pontos de intersecção entre dois segmentos perpendiculares, ao montar o cubo, coincidem com algum dos vértices destacados) e as doze arestas são os segmentos de reta mais espessos e que são lados dos quadrados da harmonia geométrica de Filolao.

\section{Pappus, construções geométricas e médias}

O grego Pappus de Alexandria, que viveu no século IV, é descrito como um matemático habilidoso, elegante e com muitas ideias. Viveu em uma época em que a matemática grega estava em declínio, mas isto não o impediu de, no ano 320 aproximadamente, escrever sua obra Coleção Matemática, composta por oito livros.

Focando nossa atenção no livro III da Coleção Matemática, onde Pappus trata da teoria das médias, vemos que ele oferece ao leitor uma construção geométrica simples, porém elegante e muito interessante, onde representa geometricamente em um semicírculo ao mesmo tempo a média aritmética, a média geométrica e a média harmônica entre dois números positivos que são representados por medidas de segmentos de reta. É esta construção geométrica o tema central do problema 3 disposto a seguir.

Problema 3. (Construção geométrica das médias por Pappus) Prove que os segmentos OD, $B D$ e FD construídos conforme orientações a seguir possuem, respectivamente, medidas iguais à média aritmética, geométrica e harmônica dos números dados por $\overline{A B} e \overline{B C}$. A construção geométrica a ser feita começa ao traçar uma reta suporte te nela representar dois segmentos de reta adjacentes, cujas medidas são os números $\overline{A B}$ e $\overline{B C}$ aos quais se quer obter as médias aritmética, geométrica e harmônica, como se vê na figura a seguir. Trace o ponto médio $O$ do segmento $A C$ assim construído e, com o compasso centrado em $O$ e com a outra extremidade do compasso em $A$, trace o semicírculo de diâmetro AC. Por B, trace uma perpendicular ao segmento $A C$, que intersectará o semicírculo em um ponto que denotaremos por $D$. Trace o segmento $O D$, cuja medida é o raio do semicírculo construído. Para finalizar, trace por B uma perpendicular ao segmento $O D$ e denote por $F$ o ponto de intersecção desta perpendicular com o segmento $O D$.

Demonstração. A figura 2 visualiza o que se passa. Inicialmente, provemos que $\overline{O D}$ é a média aritmética de $\overline{A B}$ E $\overline{B C}$. Temos que $\overline{O D}=r$, onde $r$ é o raio do semicírculo construído conforme descrito acima; e claramente $\overline{A B}+\overline{B C}=\overline{A C}=2 r$, de modo que $\overline{A B}+\overline{B C}=2 \overline{O D}$, $\log 0$

$$
\overline{O D}=\frac{\overline{A B}+\overline{B C}}{2}
$$

e portanto $\overline{O D}$ é a média aritmética de $\overline{A B}$ e $\overline{B C}$.

Para ver que $\overline{B D}$ é a média geométrica de $\overline{A B}$ e $\overline{B C}$, note que o triângulo $A D C$ está inscrito no semicírculo que foi construído e portanto o triângulo $A D C$ é retângulo em $D$. Sua altura relativa à base $A C$, que é o segmento $B D$, satisfaz $\overline{B D}^{2}=\overline{A B} \cdot \overline{B C}$, pelas relações trigonométricas existentes em um triângulo retângulo. Logo,

$$
\overline{B D}=\sqrt{\overline{A B} \cdot \overline{B C}}
$$

e assim $\overline{B D}$ é a média geométrica de $\overline{A B}$ e $\overline{B C}$.

Por fim, provemos que $\overline{F D}$ é a média harmônica de $\overline{A B}$ e $\overline{B C}$. Para tanto, note que os triângulos $D F B$ e $D B O$ 


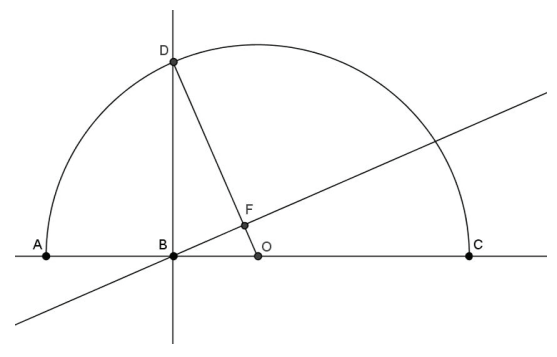

Figura 2: Semicírculo de Pappus e as médias.

são semelhantes, visto que o ângulo com vértice em $D$ é ângulo comum a estes triângulos; ainda, estes triângulos $D F B$ e $D B O$ possuem, respectivamente, ângulo reto com vértice em $F$ e $B$. Então, desta relação de semelhança, temos que

$$
\frac{\overline{F D}}{\overline{B D}}=\frac{\overline{B D}}{\overline{O D}}
$$

e assim

$$
\overline{F D}=\frac{\overline{B D}^{2}}{\overline{O D}} .
$$

Como vimos acima que $\overline{B D}^{2}=\overline{A B} \cdot \overline{B C}$, segue que

$$
\overline{F D}=\frac{\overline{A B} \cdot \overline{B C}}{\overline{O D}} .
$$

Vimos também que $\overline{O D}=\frac{\overline{A B}+\overline{B C}}{2}$ quando provamos que $\overline{O D}$ é a média aritmética de $\overline{A B}$ e $\overline{B C}$ e portanto temos

$$
\begin{gathered}
\overline{F D}=\frac{\overline{A B} \cdot \overline{B C}}{\frac{\overline{A B}+\overline{B C}}{2}} \\
\overline{F D}=\frac{2 \cdot \overline{A B} \cdot \overline{B C}}{\overline{A B}+\overline{B C}},
\end{gathered}
$$

o que comprova o último resultado a ser demonstrado.

Problema 4. Aproveitando a construção geométrica realizada no problema 3, prove a desigualdade das médias: dados dois números, a média aritmética entre eles é sempre maior ou igual do que sua média geométrica, que por sua vez é sempre maior ou igual do que sua média harmônica.

Demonstração. Se $\overline{A B}=\overline{B C}$, o que só ocorre se, e somente se, $B$ e $O$ são pontos coincidentes, trivialmente verificamos que as médias são iguais entre si. Se $\overline{A B} \neq \overline{B C}$, observe que no triângulo retângulo $D B O$ temos que $\overline{O D}>\overline{B D}$, visto que em qualquer triângulo retângulo a hipotenusa é sempre maior que qualquer um dos seus catetos. Analogamente, no triângulo retângulo $D B F$ temos que $\overline{B D}>\overline{F D}$. Destas duas últimas desigualdades, temos que

$$
\overline{O D}>\overline{B D}>\overline{F D}
$$

e assim concluímos que, dados dois números positivos, aqui representados por $\overline{A B}$ e $\overline{B C}$, a média aritmética entre eles é sempre maior ou igual do que sua média geométrica, e esta por sua vez é sempre maior ou igual do que sua média harmônica.

O livro III da Coleção Matemática traz ainda outra construção elegante, desta vez exclusiva para obtenção da média harmônica. Esta construção geométrica da média harmônica será vista agora, no problema 5.

Problema 5. (Construção geométrica da média harmônica por Pappus). Prove que a medida do segmento OC, construído conforme as instruções a seguir, é a média harmônica dos números dados pelas medidas dos segmentos $O A$ e OB. A construção geométrica a ser feita é a seguinte: dada uma reta suporte $t$, fixe nela um ponto $O$ e represente na mesma semirreta os pontos $A$ e $B$, de modo que $O A$ e $O B$ tenham como medidas os números para os quais se quer saber a média harmônica. Sem perda de generalidade, seja $\overline{O B}>\overline{O A}$, como indica a figura a seguir. Trace por $B$ uma perpendicular à reta te nela marque os pontos $D$ e $E$, de modo que $\overline{B D}=\overline{B E}=k$, onde $k$ é um número real positivo qualquer. Trace então o segmento OD. Em seguida, trace outra perpendicular à reta t, agora passando por A e seja F o ponto de intersecção entre esta última perpendicular e o segmento OD. Por fim, trace o segmento EF e seja C o ponto de intersecção dos segmentos $O B$ e EF.

Demonstração. A figura 3 nos permite visualizar o que ocorre. Os triângulos $O A F$ e $O B D$ são semelhantes, visto que o ângulo com vértice no ponto $O$ é comum a ambos; ainda, o triângulo $O A F$ tem no seu vértice $A$ um ângulo reto, o mesmo ocorrendo no triângulo $O B D$ em seu vértice $B$. Desta semelhança de triângulos temos que

$$
\frac{\overline{O A}}{\overline{O B}}=\frac{\overline{A F}}{\overline{B D}} .
$$

Como $\overline{B D}=\overline{B E}$, segue que

$$
\frac{\overline{O A}}{\overline{O B}}=\frac{\overline{A F}}{\overline{B E}} .
$$

Notemos agora que os triângulos $A C F$ e $B C E$ são também semelhantes entre si, visto que possuem um ângulo oposto pelo vértice em $C$ e, ainda, o triângulo $A C F$ tem no seu vértice $A$ um ângulo reto, o que também ocorre no triângulo $B C E$ em relação ao seu vértice $B$. Desta outra semelhança de triângulos temos que

$$
\frac{\overline{A C}}{\overline{C B}}=\frac{\overline{A F}}{\overline{B E}} .
$$

Como $\overline{A C}=\overline{O C}-\overline{O A}$ e $\overline{C B}=\overline{O B}-\overline{O C}$, segue que

$$
\frac{\overline{O C}-\overline{O A}}{\overline{O B}-\overline{O C}}=\frac{\overline{A F}}{\overline{B E}} .
$$




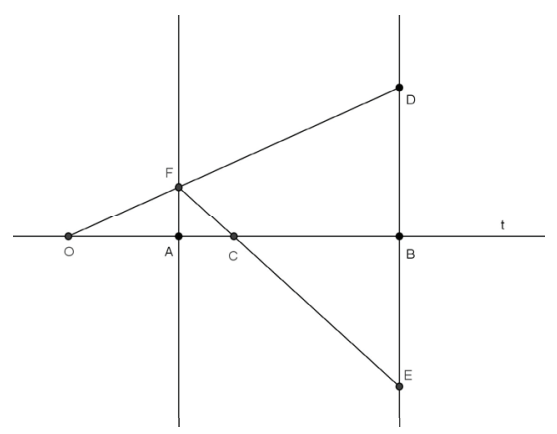

Figura 3: Construção geométrica da média harmônica por Pappus.

Das igualdades (2) e (3) temos que

$$
\frac{\overline{O A}}{\overline{O B}}=\frac{\overline{O C}-\overline{O A}}{\overline{O B}-\overline{O C}} .
$$

Pela propriedade fundamental das proporções, temos

$$
\begin{aligned}
\overline{O B} \cdot(\overline{O C}-\overline{O A}) & =\overline{O A} \cdot(\overline{O B}-\overline{O C}) \\
\overline{O B} \cdot \overline{O C}-\overline{O A} \cdot \overline{O B} & =\overline{O A} \cdot \overline{O B}-\overline{O A} \cdot \overline{O C} \\
\overline{O B} \cdot \overline{O C}+\overline{O A} \cdot \overline{O C} & =\overline{O A} \cdot \overline{O B}+\overline{O A} \cdot \overline{O B} \\
\overline{O C}(\overline{O B}+\overline{O A}) & =2 \cdot \overline{O A} \cdot \overline{O B}
\end{aligned}
$$

e assim, dividindo ambos os membros desta última igualdade por $\overline{O B}+\overline{O A} \neq 0$, temos

$$
\overline{O C}=\frac{2 \cdot \overline{O A} \cdot \overline{O B}}{\overline{O A}+\overline{O B}} .
$$

Assim, demonstramos que a medida do segmento $O C$, construído como orientou o grande matemático grego Pappus de Alexandria, é a média harmônica das medidas dos segmentos $O A$ e $O B$.

\section{Problemas e aplicações com média e progressão harmônica}

Mesmo quando restrita a apenas três termos desconhecidos, problemas com progressões harmônicas se revelam interessantes; vejamos alguns desses problemas.

Problema 6. Se $a^{2}, b^{2}$ e c $c^{2}$ estão em progressão aritmética, então $b+c, a+c$ e $a+b$ estão em progressão harmônica.

Solução. Se $a=b=c$ a progressão aritmética $\left(a^{2}, b^{2}, c^{2}\right)$ é estacionária, bem como a sequência $(b+c, a+c, a+b)$, que trivialmente se vê neste caso que é uma progressão harmônica.

Caso contrário, como $a^{2}, b^{2}$ e $c^{2}$ formam uma progressão aritmética, então existe uma constante real $r$ tal que $c^{2}-b^{2}=r, c^{2}-a^{2}=2 r$ e $b^{2}-a^{2}=r$.
De $c^{2}-b^{2}=r$, temos $(c-b)(b+c)=r$ e, dividindo ambos os lados desta última igualdade por $c-b$, o que é possível pois $b \neq c$, temos $\frac{b+c}{1}=\frac{r}{c-b}$. Segue que

$$
\frac{1}{b+c}=\frac{c-b}{r} \text {. }
$$

Analogamente, de $c^{2}-a^{2}=2 r$ e $b^{2}-a^{2}=r$ temos, respectivamente, que

$$
\frac{1}{a+c}=\frac{c-a}{2 r}
$$

e

$$
\frac{1}{a+b}=\frac{b-a}{r} \text {. }
$$

Provemos que os números $\frac{1}{b+c}, \frac{1}{a+c}, \frac{1}{a+b}$, nesta ordem, estão em progressão aritmética.

Subtraindo a igualdade (4) da igualdade (5), temos que

$$
\frac{1}{a+c}-\frac{1}{b+c}=\frac{2 b-a-c}{2 r} .
$$

Analogamente, subtraindo a igualdade (5) da igualdade (6), temos

$$
\frac{1}{a+b}-\frac{1}{a+c}=\frac{2 b-a-c}{2 r} .
$$

Logo, das duas últimas igualdades, temos que

$$
\frac{1}{a+c}-\frac{1}{b+c}=\frac{1}{a+b}-\frac{1}{a+c}
$$

e portanto

$$
\frac{1}{b+c}, \frac{1}{a+c}, \frac{1}{a+b}
$$

formam nesta ordem uma progressão aritmética, visto que a diferença entre o segundo e o primeiro termo é igual à diferença entre o terceiro e o segundo termo. Por definição, segue que os números $b+c, a+c$ e $a+b$ estão em progressão harmônica.

Problema 7. Se a, b, c estão em progressão harmônica, então ocorre o mesmo com $\frac{a}{b+c}, \frac{b}{a+c}, \frac{c}{a+b}$.

Solução. Sejam $m=\frac{a}{b+c}$ e $n=\frac{c}{a+b}$. Sabemos que interpolar um meio harmônico $k$ entre $m$ e $n$ equivale a formar a progressão harmônica $(m, k, n)$. Como $k$ deve ser média harmônica de $m$ e $n$, temos que $k=\frac{2 m n}{m+n}$, logo:

$$
k=\frac{2\left(\frac{a}{b+c}\right)\left(\frac{c}{a+b}\right)}{\frac{a}{b+c}+\frac{c}{a+b}}
$$


portanto, desenvolvendo esta expressão segue que

$$
\begin{aligned}
& k=\frac{\frac{2 a c}{(b+c)(a+b)}}{\frac{a(a+b)+c(b+c)}{(b+c)(a+b)}} \\
& k=\frac{2 a c}{a(a+b)+c(b+c)}
\end{aligned}
$$

e assim temos que

$$
k=\frac{2 a c}{a^{2}+b(a+c)+c^{2}} .
$$

Por outro lado, como $(a, b, c)$ por hipótese é progressão harmônica, então por definição temos que $\left(\frac{1}{a}, \frac{1}{b}, \frac{1}{c}\right)$ é uma progressão aritmética. Então, vale a igualdade:

$$
\frac{1}{c}-\frac{1}{b}=\frac{1}{b}-\frac{1}{a}
$$

e então, temos

$$
\begin{gathered}
\frac{1}{b}+\frac{1}{b}=\frac{1}{a}+\frac{1}{c} \\
\frac{2}{b}=\frac{a+c}{a c} .
\end{gathered}
$$

Portanto, segue que

$$
b(a+c)=2 a c .
$$

Substituindo a igualdade (8) tanto no numerador quanto no denominador da expressão à direita da igualdade (7), obtemos

$$
k=\frac{b(a+c)}{a^{2}+2 a c+c^{2}}
$$

e assim

$$
k=\frac{b(a+c)}{(a+c)^{2}} .
$$

Note que $(a, b, c)$ estão em progressão harmônica, então temos que $b=\frac{2 a c}{a+c}$ e $\operatorname{assim} a+c=\frac{2 a c}{b}$. Mas, por definição, a progressão harmônica dada tem seus termos $a, b, c$ todos diferentes de zero, $\log a+c=\frac{2 a c}{b} \neq 0$. Dividindo o numerador e o denominador da expressão à direita da igualdade (9) acima por $a+c$, obtemos que $k=\frac{b}{a+c}$. Logo, $(m, k, n)=\left(\frac{a}{b+c}, \frac{b}{a+c}, \frac{c}{a+b}\right)$ é uma progressão harmônica.

Problema 8. Sejam $a, b$ e c números reais positivos que nesta ordem formam uma progressão harmônica. Se $a, b$ e $c$ representam, respectivamente, o lado, a área da face e o volume de um cubo, demonstre que este cubo é o cubo unitário.
Solução. Temos que $b=a^{2}$ e $c=a^{3}, \operatorname{logo}\left(a, a^{2}, a^{3}\right)$ é uma progressão harmônica e então $a^{2}$ é a média harmônica de $a$ e $a^{3}$. Assim, $a^{2}=\frac{2 a \cdot a^{3}}{a+a^{3}}$ e segue que

$$
2 a^{4}=a^{3}+a^{5} \text {. }
$$

Como $a$ é positivo, dividindo a expressão acima por $a^{3}$ obtemos:

$$
\begin{gathered}
2 a=1+a^{2} \\
a^{2}-2 a+1=0 \\
(a-1)^{2}=0
\end{gathered}
$$

e assim $a=-1$ ou $a=1$. Novamente, lembremos que $a$ é positivo e portanto $a=-1$ não é resposta deste problema. Consequentemente, o cubo tem lado $a=1$, área da face $a^{2}=1^{2}=1$ e volume igual $a^{3}=1^{3}=1$, o que comprova que o cubo dado é o cubo unitário.

Finalizamos esta seção com uma aplicação que mostra que utilizamos a média harmônica quando lidamos com grandezas inversamente proporcionais como velocidade e tempo, por exemplo. Mais especificamente, mantendo fixa a distância total percorrida, a média harmônica das velocidades deste corpo em todos os trechos parciais da distância total percorrida é igual à velocidade média do corpo em todo percurso, conforme veremos no problema 9 a seguir para o caso de dois trechos.

Problema 9. Um carro viaja à razão de $r_{1}$ quilômetros por hora de $A$ até $B$ e então retorna de $B$ a $A$ à razão de $r_{2}$ quilômetros por hora. Mostre que a velocidade média do percurso de ida e volta é a média harmônica de $r_{1}$ e $r_{2}$.

Solução. Sejam $d, t_{1}$ e $t_{2}$, respectivamente, a distância total percorrida, o tempo da viagem de $A$ até $B$ e o tempo da viagem de $B$ a $A$.

Temos que $t_{1}=\frac{d}{2 r_{1}}$ e $t_{2}=\frac{d}{2 r_{2}}$. A velocidade média $r_{m}$ deste carro é tal que $r_{m}=\frac{d}{t_{1}+t_{2}}$, logo segue que:

$$
r_{m}=\frac{d}{\frac{d}{2 r_{1}}+\frac{d}{2 r_{2}}}
$$

Como $d \neq 0$, dividindo a igualdade acima por $d$ obtemos:

e portanto

$$
\begin{gathered}
r_{m}=\frac{1}{\frac{1}{2 r_{1}}+\frac{1}{2 r_{2}}} \\
r_{m}=\frac{1}{\frac{r_{2}}{2 r_{1} r_{2}}+\frac{r_{1}}{2 r_{1} r_{2}}}
\end{gathered}
$$

$$
r_{m}=\frac{2 r_{1} r_{2}}{r_{1}+r_{2}}
$$

logo a velocidade média $r_{m}$ do percurso de ida e volta é a média harmônica de $r_{1}$ e $r_{2}$. 


\section{A série harmônica}

A série harmônica, aqui denominada $S_{h}$, é a soma infinita dos inversos dos números inteiros positivos, isto é:

$$
S_{h}=\frac{1}{1}+\frac{1}{2}+\frac{1}{3}+\frac{1}{4}+\frac{1}{5}+\frac{1}{6}+\ldots
$$

Se uma série tem soma finita, então esta série é dita convergente; caso contrário, a série é dita divergente. Veremos aqui que a série harmônica é divergente.

Percebe-se facilmente que os termos da série harmônica decrescem tendendo para zero, dando a impressão de que esta série é igual a uma constante. De fato, somando os primeiros trinta milhões de termos da série harmônica o resultado é um número menor que 18. Mas esta impressão de convergência não se confirma, e o matemático francês Nicole Oresme $(1325$ - 1382) foi o primeiro a provar tal fato.

Nicole Oresme é considerado por alguns historiadores como o maior matemático do século XIV. Acredita-se que Oresme seja precursor da geometria analítica, importante ramo da matemática estudada no ensino médio. Ao lidar com a série de Suiseth, Oresme também se revela como precursor da análise infinitesimal, ramo de muita importância da matemática de nível superior. Conforme afirmado anteriormente, Oresme teve sucesso ao demonstrar que a série harmônica é divergente, o que nos revela o quanto é fundamental a utilização do raciocínio lógico para verificar algo que é impossível constatar de outro modo.

A série harmônica diverge, pois sendo $k$ um número natural fixo porém arbitrário com $k \geq 2$, como temos $2^{k-1}+m \leq 2^{k}$, para todo $m$ número natural tal que $1 \leq m \leq 2^{k-1}$, segue que $\frac{1}{2^{k-1}+m} \geq \frac{1}{2^{k}} ;$ assim ao agruparmos todos os seus termos da forma $\frac{1}{2^{k-1}+1}$ até $\frac{1}{2^{k}}$, para todo $k \geq 2$, obtemos:

$$
\begin{aligned}
& S_{h}=\frac{1}{1}+\frac{1}{2}+\left(\frac{1}{3}+\frac{1}{4}\right)+\left(\frac{1}{5}+\cdots+\frac{1}{8}\right)+\ldots \\
& S_{h} \geq \frac{1}{1}+\frac{1}{2}+\left(\frac{1}{4}+\frac{1}{4}\right)+\left(\frac{1}{8}+\cdots+\frac{1}{8}\right)+\ldots
\end{aligned}
$$

Notando que cada um dos parênteses acima é igual a $\frac{1}{2}$, pois a soma de $2^{k-1}$ parcelas iguais de $\frac{1}{2^{k}}$ é igual a $\frac{2^{k-1}}{2^{k}}=\frac{1}{2}$, segue que:

$$
S_{h} \geq 1+\frac{1}{2}+\frac{1}{2}+\frac{1}{2}+\ldots
$$

Como a série $1+\frac{1}{2}+\frac{1}{2}+\frac{1}{2}+\ldots$ é claramente divergente, uma vez que podemos somar infinitas parcelas iguais a $\frac{1}{2}$, consequentemente a série harmônica diverge.
A retirada de uma quantidade finita de termos da série harmônica obviamente não fará a mesma convergir. Entretanto, provaremos que uma retirada infinita de termos da série harmônica pode implicar na convergência da série remanescente. A ideia que será exposta é válida para qualquer número formado por um único algarismo.

Consideremos a série que denominaremos por $S_{7}$, formada pela série harmônica menos todos os elementos que possuem o algarismo 7 no denominador; assim, $\frac{1}{7}, \frac{1}{37}, \frac{1}{71}$ e $\frac{1}{60738}$ são exemplos de termos que não fazem parte de $S_{7}$. Temos que:

$$
S_{7}=\frac{1}{1}+\cdots+\frac{1}{6}+\frac{1}{8}+\cdots+\frac{1}{16}+\frac{1}{18}+\ldots
$$

Cada um dos 8 primeiros termos, onde os denominadores possuem apenas um algarismo, é menor ou igual a 1 e assim

$$
\frac{1}{1}+\cdots+\frac{1}{6}+\frac{1}{8}<8
$$

Analogamente, cada um dos $8 \cdot 9$ termos seguintes, onde os denominadores possuem somente dois algarismos, é menor ou igual a $\frac{1}{10}$ e portanto a soma destes termos é menor que $8 \cdot 9 \cdot \frac{1}{10}=8 \cdot \frac{9}{10}$.

O mesmo raciocínio vale para cada um dos $8 \cdot 9$. 9 termos seguintes, onde os denominadores possuem exatos três algarismos, é menor ou igual a $\frac{1}{100}$ e portanto a soma destes termos é menor que $8 \cdot 9^{2} \cdot \frac{1}{100}=8 \cdot \frac{9^{2}}{10^{2}}$.

Prosseguindo este raciocínio para os demais termos da série $S_{7}$, vemos que

$$
S_{7}<8+8 \cdot \frac{9}{10}+8 \cdot \frac{9^{2}}{10^{2}}+\ldots
$$

Então, como $8+8 \cdot \frac{9}{10}+8 \cdot \frac{9^{2}}{10^{2}}+\ldots$ é a soma dos infinitos termos de uma progressão geométrica tal que $a_{1}=8$ e razão $q=\frac{9}{10}$, segue que

$$
8+8 \cdot \frac{9}{10}+8 \cdot \frac{9^{2}}{10^{2}}+\cdots=\frac{8}{1-\frac{9}{10}}=80
$$

e assim $S_{7}<80$, logo $S_{7}$ converge.

Agora, uma interessante discussão sobre o motivo da série ser denominada de harmônica. Inicialmente, note que a média harmônica de dois números dados é igual ao inverso da média aritmética dos seus inversos. De fato, por definição de média harmônica, temos que dados $a$ e $b$, sua média harmônica $H$ é tal que $\frac{1}{a}+\frac{1}{b}=\frac{1}{H}+\frac{1}{H}=\frac{2}{H^{\prime}}$ logo:

$$
\frac{1}{\frac{1}{a}+\frac{1}{b}}=\frac{H}{2} \text {. }
$$


Multiplicando por 2 a expressão acima, temos

$$
\frac{2}{\frac{1}{a}+\frac{1}{b}}=H
$$

e portanto

$$
H=\frac{1}{\frac{\frac{1}{a}+\frac{1}{b}}{2}} .
$$

A série harmônica tem esse nome pois seu termo geral $\frac{1}{n}$ é igual à média harmônica do seu antecessor $\frac{1}{n-1}$ e seu sucessor $\frac{1}{n+1}$. Com efeito, como vimos antes, a média harmônica de dois números dados é igual ao inverso da média aritmética dos seus inversos; tomando os inversos do antecessor e do sucessor de $\frac{1}{n}$, dados por $n-1$ e $n+1$, respectivamente, a média aritmética entre eles é igual a $\frac{(n-1)+(n+1)}{2}=n$, cujo inverso é $\frac{1}{n}$, que é o termo geral da série harmônica. Isso explica o motivo para a denominação série harmônica, mas é natural perguntar agora o motivo da média harmônica ter esse nome.

A origem é incerta, porém historiadores acreditam que o mais provável é que os pitagóricos tenham assim a denominado. Há registros de que dois pitagóricos, Arquitas e Hipaso, tenham mudado o nome da média de subcontrária, designação inicial, para a atual, harmônica. Outras possibilidades para a origem, bem remotas, são o Egito e a Babilônia. Mas o motivo da denominação é conhecido: a média harmônica aparece na consideração dos tons de um monocórdio. O dicionarista Aurélio Buarque de Holanda Ferreira define o monocórdio como o instrumento composto de uma caixa de ressonância, sobre a qual se estende uma corda que fica apoiada sobre dois cavaletes móveis, e que já era usado no tempo de Pitágoras para o estudo e cálculo das relações entre as vibrações sonoras.

A matemática explica melhor o que ocorre. Considere três tons sonoros diferentes de um monocórdio, o primeiro produzido quando a corda é esticada em todo seu tamanho, aqui considerado 1 unidade de comprimento; a oitava, que é quando a corda é reduzida à $\frac{1}{2}$ de comprimento; e a quinta, que é quando a corda tem $\frac{2}{3}$ de comprimento. A média harmônica $H$ dos números 1 e $\frac{1}{2}$ é, igual a:

$$
H=\frac{2 \cdot 1 \cdot \frac{1}{2}}{1+\frac{1}{2}}=\frac{2}{3}
$$

Então, $\frac{2}{3}$ é a média harmônica de 1 e $\frac{1}{2}$, de modo que a frequência da quinta é a média harmônica das outras duas frequências. A palavra harmônica tem a ver, portanto, com a harmonia do som intermediário relativo aos outros dois sons.

Isso explica também a proporção musical: dados dois inteiros positivos $a$ e $b$, os pitagóricos descobriram uma importante relação, chamada por eles de proporção musical, entre $a, b$, sua média aritmética $A$ e sua média harmônica $H$, expressa por $\frac{a}{A}=\frac{H}{b}$.

Encerramos esta seção verificando a validade da proporção musical. Para tal, calculemos a média harmônica $H$ por esta proporção; então, devemos encontrar como resultado $H=\frac{2 a b}{a+b}$. Temos $A=\frac{a+b}{2}$, logo segue que:

$$
\begin{aligned}
& \frac{a}{\frac{a+b}{2}}=\frac{H}{b} \\
& \frac{2 a}{a+b}=\frac{H}{b}
\end{aligned}
$$

e assim obtemos

$$
H=\frac{2 a b}{a+b},
$$

comprovando a validade da proporção musical.

\section{Progressão harmônica de segunda ordem e o triângulo harmônico}

Do mesmo modo como se define a progressão aritmética de ordem $k$, podemos definir a progressão harmônica de ordem $k$. Concentraremos a atenção na ordem dois, isto é, abordaremos a progressão harmônica de segunda ordem.

Definição 7.1. Uma progressão harmônica de ordem $k$ é uma sequência finita ou infinita de números, todos diferentes de zero, tais que seus inversos formam uma progressão aritmética de ordem $k$.

Então, tomando $k=2$, temos que uma progressão harmônica de segunda ordem é uma sequência finita ou infinita de números, todos diferentes de zero, tais que seus inversos formam uma progressão aritmética de segunda ordem.

Seja $\left(h_{n}\right)$ uma progressão harmônica de segunda ordem qualquer e $\left(a_{n}\right)$, a progressão aritmética de segunda ordem associada a $\left(h_{n}\right)$. Ainda, seja $\left(b_{n}\right)$ a progressão aritmética de primeira ordem de razão $r$ associada a $\left(a_{n}\right)$. Como o termo geral da progressão aritmética de segunda ordem é expresso por

$$
a_{n}=a_{1}+b_{1}(n-1)+\frac{(n-1)(n-2) r}{2},
$$

segue que

$$
h_{n}=\frac{1}{a_{1}+b_{1}(n-1)+\frac{(n-1)(n-2) r}{2}} .
$$


O problema a seguir é facilmente resolvido com o uso da igualdade (10).

Problema 10. Considere $\left(a_{n}\right)$ a progressão aritmética de segunda ordem expressa por $\left(a_{n}\right)=(2,6,12,20, \ldots)$, a qual está associada a progressão aritmética de primeira or$\operatorname{dem}\left(b_{n}\right)=(4,6,8,10, \ldots)$. Determine o termo geral da progressão harmônica de segunda ordem $\left(h_{n}\right)$ associada a $\left(a_{n}\right)$, bem como seu sétimo termo.

Solução. Temos $a_{1}=2, b_{1}=4$ e a razão $r=6-4=2$. Pela igualdade (10), segue que

$$
h_{n}=\frac{1}{2+4(n-1)+\frac{(n-1)(n-2) 2}{2}} .
$$

Desenvolver algebricamente esta última expressão resulta em

$$
h_{n}=\frac{1}{2+4 n-4+n^{2}-3 n+2}
$$

e portanto o termo geral $h_{n}$ é dado por

$$
h_{n}=\frac{1}{n^{2}+n} \text {. }
$$

De posse da igualdade (11), vemos sem dificuldade que o sétimo termo de $\left(h_{n}\right)$ é tal que

$$
h_{7}=\frac{1}{7^{2}+7}=\frac{1}{56} \text {. }
$$

O notável matemático alemão Gottfried Wilhelm Leibniz (1646 - 1716) é, às vezes, considerado o último sábio a conseguir conhecimento universal, por ter estudado matemática, direito, teologia e filosofia na universidade. Ele contribuiu significativamente nestas áreas e também em história, diplomacia, política e metafísica. Em matemática, Leibniz é considerado personagem principal no descobrimento do cálculo diferencial e integral, área da matemática de nível superior. Começou a produzir em matemática quando estudou séries e, em especial, calculou várias séries através do triângulo harmônico.

O triângulo harmônico que analisaremos agora (veja figura 4) e que fascinou Leibniz é da seguinte forma: na primeira coluna, de cima para baixo, são escritos os termos da série harmônica, do maior para o menor; na segunda coluna, cada elemento é a diferença entre o elemento imediatamente acima e o elemento ao lado, ambos da coluna à esquerda mais próxima; para as demais colunas, o mesmo raciocínio da segunda coluna se aplica. A figura a seguir mostra os primeiros elementos do triângulo harmônico.

Por exemplo, o terceiro elemento da segunda coluna é $\frac{1}{12}$, pois $\frac{1}{2}-\frac{1}{6}=\frac{1}{12}$. Repare que $\frac{1}{2}$ e $\frac{1}{6}$ são os elementos que estão, respectivamente, imediatamente acima e

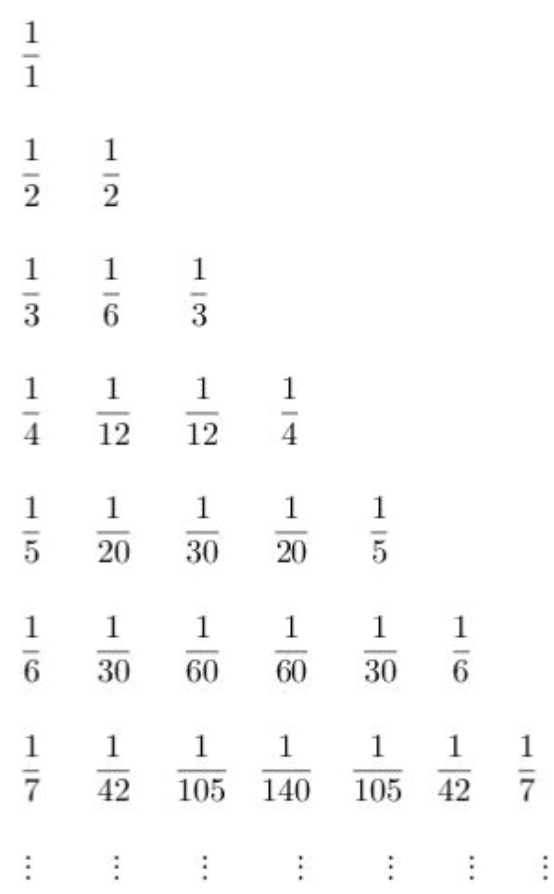

Figura 4: Triângulo harmônico.

ao lado da primeira coluna, que é a coluna da esquerda mais próxima em relação à segunda coluna.

Analisemos o triângulo harmônico; para tanto, denotemos por $k$ a posição da coluna do triângulo harmônico, da esquerda para a direita; e denotemos por $n$, a posição, considerada de cima para baixo, do elemento localizado na k-ésima coluna do triângulo harmônico. Assim, um elemento qualquer do triângulo harmônico pode ser denotado por $T_{n, k}$. Por exemplo, $T_{2,3}$ denota o segundo elemento da terceira coluna do triângulo harmônico, conforme se pode ver na figura 5 a seguir.

Com esta notação, os termos do triângulo harmônico são, por definição, tais que

$$
T_{n, k}=T_{n, k-1}-T_{n+1, k-1} .
$$

A proposição 7.2 permite calcular um termo qualquer do triângulo harmônico somente em função de $n$ e $k$.

Proposição 7.2. Seja $T_{n, k}$ um elemento qualquer do triângulo harmônico. Temos que

$$
T_{n, k}=\frac{1}{n} \cdot \frac{1}{\left(\begin{array}{c}
n+k-1 \\
n
\end{array}\right)}
$$

Demonstração. Provemos por indução em $k$, deixando $n$ fixo porém arbitrário. Para $k=1$, temos que:

$$
\begin{gathered}
T_{n, 1}=\frac{1}{n} \cdot \frac{1}{\left(\begin{array}{c}
n+1-1 \\
n
\end{array}\right)} \\
T_{n, 1}=\frac{1}{n} \cdot \frac{1}{\left(\begin{array}{l}
n \\
n
\end{array}\right)}
\end{gathered}
$$




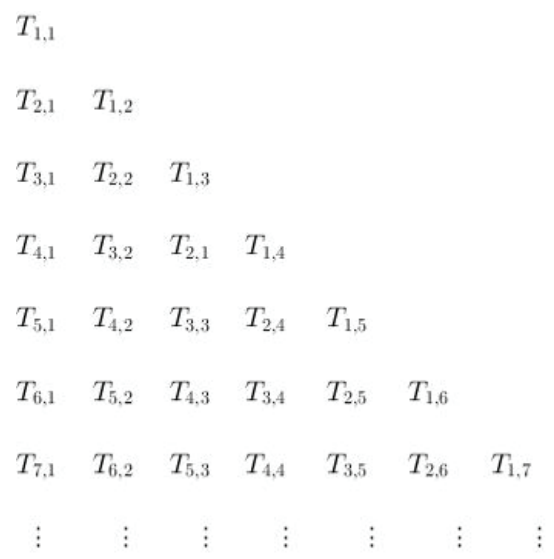

Figura 5: Triângulo harmônico conforme notação usada.

e, como $\left(\begin{array}{l}n \\ n\end{array}\right)=1$, segue que

$$
T_{n, 1}=\frac{1}{n} \cdot \frac{1}{1}=\frac{1}{n}
$$

verificando assim a validade do caso $k=1$, pois os elementos da primeira coluna são os termos da série harmônica, que são da forma $\frac{1}{n}$.

Se, para algum número natural $k$ temos válida a igualdade $T_{n, k}=\frac{1}{n} \cdot \frac{1}{\left(\begin{array}{c}n+k-1 \\ n\end{array}\right)}$, então basta mostrar que é válida também a igualdade $T_{n, k+1}=\frac{1}{n} \cdot \frac{1}{\left(\begin{array}{c}n+k \\ n\end{array}\right)}$, para que a proposição fique totalmente demonstrada.

Temos, pela igualdade (12), que

$$
T_{n, k}=T_{n, k-1}-T_{n+1, k-1},
$$

$\log 0$

$$
T_{n, k+1}=T_{n, k}-T_{n+1, k}
$$

Como por hipótese de indução temos que

$$
T_{n, k}=\frac{1}{n} \cdot \frac{1}{\left(\begin{array}{c}
n+k-1 \\
n
\end{array}\right)}
$$

também temos que

$$
T_{n+1, k}=\frac{1}{n+1} \cdot \frac{1}{\left(\begin{array}{c}
n+1+k-1 \\
n+1
\end{array}\right)}=\frac{1}{n+1} \cdot \frac{1}{\left(\begin{array}{l}
n+k \\
n+1
\end{array}\right)},
$$

logo dessas duas últimas igualdades e também de (13) segue que

$$
T_{n, k+1}=\frac{1}{n} \cdot \frac{1}{\left(\begin{array}{c}
n+k-1 \\
n
\end{array}\right)}-\frac{1}{n+1} \cdot \frac{1}{\left(\begin{array}{l}
n+k \\
n+1
\end{array}\right)} .
$$

A definição de número binomial, aplicada na igualdade (14), nos permite afirmar que

$$
T_{n, k+1}=\frac{1}{n} \cdot \frac{1}{\frac{(n+k-1) !}{n !(k-1) !}}-\frac{1}{n+1} \cdot \frac{1}{\frac{(n+k) !}{(n+1) !(k-1) !}} .
$$

Prosseguindo com os cálculos algébricos temos:

$$
T_{n, k+1}=\frac{1}{n} \cdot \frac{1}{\frac{(n+k) !}{n ! k !}} \cdot \frac{n+k}{k}-\frac{1}{n} \cdot \frac{1}{\frac{(n+k) !}{n ! k !}} \cdot \frac{n}{k} .
$$

Novamente, da definição de número binomial, temos que $\frac{(n+k) !}{n ! k !}=\left(\begin{array}{c}n+k \\ n\end{array}\right)$ e assim

$$
T_{n, k+1}=\frac{1}{n} \cdot \frac{1}{\left(\begin{array}{c}
n+k \\
n
\end{array}\right)} \cdot \frac{n+k}{k}-\frac{1}{n} \cdot \frac{1}{\left(\begin{array}{c}
n+k \\
n
\end{array}\right)} \cdot \frac{n}{k} .
$$

Colocando $\frac{1}{n} \cdot \frac{1}{\left(\begin{array}{c}n+k \\ n\end{array}\right)}$ em evidência, temos

$$
\begin{gathered}
T_{n, k+1}=\frac{1}{n} \cdot \frac{1}{\left(\begin{array}{c}
n+k \\
n
\end{array}\right)}\left(\frac{n+k}{k}-\frac{n}{k}\right) \\
T_{n, k+1}=\frac{1}{n} \cdot \frac{1}{\left(\begin{array}{c}
n+k \\
n
\end{array}\right)}\left(\frac{n+k-n}{k}\right) \\
T_{n, k+1}=\frac{1}{n} \cdot \frac{1}{\left(\begin{array}{c}
n+k \\
n
\end{array}\right)}\left(\frac{k}{k}\right) \\
T_{n, k+1}=\frac{1}{n} \cdot \frac{1}{\left(\begin{array}{c}
n+k \\
n
\end{array}\right)} \cdot 1,
\end{gathered}
$$

portanto

$$
T_{n, k+1}=\frac{1}{n} \cdot \frac{1}{\left(\begin{array}{c}
n+k \\
n
\end{array}\right)}
$$

e a proposição está demonstrada por completo.

Corolário 7.3. O termo geral do triângulo harmônico pode ser expresso também como

$$
T_{n, k}=\frac{(k-1) !}{n \cdot(n+1) \ldots(n+(k-1))} .
$$

Demonstração. Basta notar que da proposição 7.2 temos $T_{n, k}=\frac{1}{n} \cdot \frac{1}{\left(\begin{array}{c}n+k-1 \\ n\end{array}\right)}, \log \mathrm{o}$

$$
\begin{gathered}
T_{n, k}=\frac{1}{n} \cdot \frac{1}{\frac{(n+(k-1)) !}{n !(k-1) !}} \\
T_{n, k}=\frac{1}{n} \cdot \frac{n !(k-1) !}{(n+(k-1)) !} \\
T_{n, k}=\frac{(n-1) !(k-1) !}{(n+(k-1)) !} \\
T_{n, k}=\frac{(k-1) !}{n \cdot(n+1) \ldots(n+(k-1))},
\end{gathered}
$$

c.q.d.

O corolário a seguir é de fundamental importância em nossos estudos nesta seção, pois relaciona o triângulo harmônico com a progressão harmônica de ordem $k$. 
Corolário 7.4. Os elementos da k-ésima coluna do triângulo harmônico formam uma progressão harmônica de ordem $k$.

Demonstração. Como sabemos que $\left(a_{n}\right)$ é uma progressão aritmética de ordem $k$ se, e somente se, $a_{n}$ pode ser dado por um polinômio de grau $k$ em $n$, então $\left(h_{n}\right)$ é uma progressão harmônica de ordem $k$ se, e somente se, $h_{n}$ pode ser expresso por uma fração de numerador igual a $1 \mathrm{e}$ denominador dado por um polinômio de grau $k$ em $n$. Sem perda de generalidade, esta afirmação permanece válida se multiplicarmos por uma constante todos os termos da progressão aritmética de ordem $k$.

Do corolário 7.3, temos que

$$
T_{n, k}=\frac{(k-1) !}{n \cdot(n+1) \ldots(n+(k-1))},
$$

isto é, $T_{n, k}$ é igual à uma fração onde o numerador é um inteiro positivo dado por $(k-1)$ ! e com denominador igual a um polinômio de grau $k$ em $n$. Segue então que os elementos da $k$-ésima coluna do triângulo harmônico formam uma progressão harmônica de ordem $k$.

A propriedade 7.5 revela que a comutatividade é válida para os índices dos termos do triângulo harmônico, como veremos a seguir.

Proposição 7.5. Sejam $T_{n, k}$ e $T_{k, n}$ termos quaisquer do triângulo harmônico. Temos que $T_{n, k}=T_{k, n}$, isto é, a troca dos indices $n$ e $k$ não altera o valor do termo.

Demonstração. Pela proposição 7.2 , temos que

$$
\mathrm{T}_{k, n}=\frac{1}{k} \cdot \frac{1}{\left(\begin{array}{c}
k+n-1 \\
k
\end{array}\right)}
$$

$\log 0$

$$
\begin{gathered}
T_{k, n}=\frac{1}{k} \cdot \frac{1}{\left(\begin{array}{c}
n+k-1 \\
k
\end{array}\right)}=\frac{1}{k} \cdot \frac{1}{\frac{(n+k-1) !}{k !(n-1) !}} \\
T_{k, n}=\frac{1}{k} \cdot \frac{1}{\frac{n(n+k-1) !}{k(k-1) ! n(n-1) !}} \\
T_{k, n}=\frac{1}{k} \cdot \frac{1}{n} \cdot \frac{1}{\frac{1}{k}} \cdot \frac{1}{\frac{(n+k-1) !}{n !(k-1) !}}
\end{gathered}
$$

$$
\begin{aligned}
& \text { Como } \frac{1}{k} \cdot \frac{1}{\frac{1}{k}}=1 \text {, segue que } \\
& T_{k, n}=\frac{1}{n} \cdot \frac{1}{\frac{(n+k-1) !}{n !(k-1) !}}=\frac{1}{n} \cdot \frac{1}{\left(\begin{array}{c}
n+k-1 \\
n
\end{array}\right)}
\end{aligned}
$$

e assim, utilizando novamente a proposição 7.2 na última igualdade aqui obtida, temos que $T_{k, n}=T_{n, k}$.
Encerramos este artigo com a maior motivação de Leibniz em relação ao triângulo harmônico: o cálculo de séries infinitas. Para entender melhor, vejamos a última proposição desta seção agora.

Proposição 7.6. A soma $S_{n, k}$ dos $n$ primeiros termos da $k$ ésima coluna, de cima para baixo, com $k>1$, é tal que

$$
S_{n, k}=\frac{1}{k-1}-\frac{(k-2) !}{(n+1) \ldots(n+(k-1))} .
$$

Demonstração. Da igualdade (12) temos que

$$
\begin{gathered}
T_{1, k}=T_{1, k-1}-T_{2, k-1} \\
T_{2, k}=T_{2, k-1}-T_{3, k-1} \\
\vdots \\
T_{n, k}=T_{n, k-1}-T_{n+1, k-1} .
\end{gathered}
$$

Somando estas $n$ igualdades membro a membro e como $S_{n, k}=T_{1, k}+T_{2, k}+\cdots+T_{n, k}$, segue que

$$
S_{n, k}=\left(T_{1, k-1}+\cdots+T_{n, k-1}\right)-\left(T_{2, k-1}+\cdots+T_{n+1, k-1}\right) .
$$

Notando que esta é uma soma telescópica, chegamos a

$$
S_{n, k}=T_{1, k-1}-T_{n+1, k-1} .
$$

Calculando agora $T_{1, k-1}$, pelo corolário 7.3 , temos que

$$
\mathrm{T}_{1, k-1}=\frac{1}{k-1}
$$

Analogamente, calculando agora $T_{n+1, k-1}$, pelo corolário 7.3, temos que

$$
T_{n+1, k-1}=\frac{(k-2) !}{(n+1) \ldots(n+(k-1))} .
$$

Substituindo as igualdades (16) e (17) na igualdade (15), obtemos que

$$
S_{n, k}=\frac{1}{k-1}-\frac{(k-2) !}{(n+1) \ldots(n+(k-1))},
$$

o que encerra a demonstração.

Voltando a falar das séries calculadas por Leibniz, observe que quando $n$ tende ao infinito, a série $S$ formada pela $k$-ésima coluna do triângulo harmônico é tal que $S=\frac{1}{k-1}$, visto que quando $n$ tende ao infinito, a parcela

$$
\frac{(k-2) !}{(n+1) \cdot(n+2) \ldots(n+(k-1))}
$$

da soma parcial $S_{n}$ tende a zero. Deste modo, Leibniz obteve o valor de convergência para infinitas séries; em particular, por exemplo, temos que

$$
\frac{1}{2}+\frac{1}{6}+\frac{1}{12}+\cdots=1,
$$


referente à soma de todos os termos da segunda coluna do triângulo harmônico; ainda, mais um exemplo, desta vez se referindo à soma de todos os termos da quinta coluna do triângulo harmônico:

$$
\frac{1}{5}+\frac{1}{30}+\frac{1}{105}+\cdots=\frac{1}{4}
$$

\section{Referências}

Antar, A. (1979). Progressões e Logaritmos, $1^{\circ}$ edn. Moderna.

Boyer, C., Merzbach, U. (1996). História da Matemática, $1^{\circ}$ edn. Edgard Blücher Ltda.

Hefez, A. (2006). Elementos de aritmética, $2^{\circ}$ edn. Sociedade Brasileira de Matemática.

Lopes, L. B. R. (1992). Manual de Sequências e Séries, $1^{\mathrm{O}}$ edn. Didática e Científica Ltda.

Lopes, L. B. R. (1998). Manual de Progressões, $1^{\circ}$ edn. Interciência.

Pólya, G. (1995). A arte de resolver problemas, $2^{\circ}$ edn. Interciência.

Strathern, P. (1998). Pitágoras e Seu Teorema em 90 Minutos, $1^{\circ}$ edn. Jorge Zahar.

Watanabe, R. G. (1996). Alergia pelo Número 7. Revista do Professor de Matemática, (31), 32. 